within and between the three successive calendar periods were also examined.

Results For patients diagnosed during 1996, 1-year survival in the most deprived group was lower than the most affluent group for 34 of the 35 cancer-sex combinations examined (27 significant at $5 \%$ ). The figure was almost identical a decade later, in 2006, with lower survival in the most deprived group for 32 of the 35 cancer-sex combinations (23 significant at $5 \%$ ). Although trends in the deprivation gap fluctuated between calendar periods, overall, between 1996 and 2006, the deprivation gap widened for 14 cancer-sex combinations (increasing by more than $2 \%$ for 3 cancers in women and 5 cancers in men), and narrowed for 21 (decreasing by more $2 \%$ for 6 cancers in women and 1 cancer in men).

Conclusion For most cancers, the deprivation gap in survival has changed very little since the introduction of the NHS Cancer Plan. Despite small reductions in the deprivation gap for some cancers, there was no obvious acceleration in the reduction since the NHS Cancer Plan. Inequalities in cancer survival were still large for many cancers in 2006

\section{LUNG CANCER INCIDENCE AND SURVIVAL IN ETHNIC GROUPS IN SOUTH-EAST ENGLAND}

doi:10.1136/jech.2010.120956.37

R H Jack, E A Davies, H Møller. King's College London, London, UK

Objective Ethnicity has not been well recorded in UK cancer registries to date. However, Hospital Episode Statistics data are available for all inpatient admissions to English NHS hospitals and include self-assigned ethnicity, which can be linked to cancer registrations. This study aimed to use all the available ethnicity data to describe the different patterns of lung cancer incidence and survival in different ethnic groups in South-East England.

Design Population data from the 2001 Census were available by age, sex, ethnic group and socio-economic deprivation (using the income quintile of the Indices of Deprivation 2000). Age- and socioeconomic deprivation-standardised incidence rate ratios were calculated for males and females in each ethnic group, using the White groups as the baseline categories. Overall survival for each ethnic group was also examined in males and females using Cox regression.

Setting South-East England population including Essex, Hertfordshire, London, Kent, Surrey and Sussex.

Participants There were 28145 male and 18257 female patients diagnosed with lung cancer resident in South-East England between 1998 and 2003. Results are presented for White, Indian, Pakistani, Bangladeshi, Black Caribbean, Black African and Chinese patients, apart from female survival results where due to small numbers the White, South Asian and Black ethnic groups were analysed.

Main outcome measures Age- and socio-economic deprivationstandardised incidence rate ratios; hazard ratios adjusted for age, socio-economic deprivation, stage of disease and treatment.

Results Compared with ethnic groups of the same sex, Bangladeshi men, White men and White women had the highest incidence rates. Differences between the White baselines and other ethnic groups were larger for women than for men. Bangladeshi men had consistently better survival estimates compared with White men (fully adjusted hazard ratio (HR) 0.46, $\mathrm{p}<0.001$ ). Indian (HR 0.84, $\mathrm{p}=0.048$ ), Black Caribbean (HR 0.87, $\mathrm{p}=0.47$ ) and Black African (HR
0.68, $\mathrm{p}=0.007)$ men also had better survival estimates. South Asian (HR 0.73, p=0.006) and Black (HR 0.74, p=0.004) women had similar better survival than White women.

Conclusions Lung cancer incidence is closely related to smoking, and prevention messages need to be targeted for different ethnic groups taking into account language and relevancy to ensure no groups are excluded. The apparent better survival of South Asian and Black patients was surprising, and more detailed follow-up studies are needed to verify these results.

\section{SURVIVAL FROM CHILDHOOD AND YOUNG ADULT CANCER IN THE NORTH OF ENGLAND, 1968-2005}

doi:10.1136/jech.2010.120956.38

${ }^{1} \mathrm{~N} O$ Basta, ${ }^{1} \mathrm{P}$ W James, ${ }^{1} \mathrm{~B}$ Gomez-Pozo, ${ }^{2} \mathrm{~A}$ W Craft, ${ }^{1} \mathrm{R}$ J 0 McNally. ${ }^{1}$ Institute of Health and Society, Newcastle University, Sir James Spence Institute, Royal Victoria Infirmary, Newcastle-upon- Tyne, UK; ${ }^{2}$ Northern Institute of Cancer Research, Newcastle University, Sir James Spence Institute, Royal Victoria Infirmary, Newcastleupon- Tyne, UK

Objective To investigate survival from cancer in children and young adults resident in the North of England.

Methods Cases aged $0-24$ years diagnosed with a primary malignancy during the period 1968-2005 were obtained from the Northern Region Young Persons' Malignant Disease Registry. Survival rates at five years were calculated using Kaplan- Meier estimation, for each diagnostic group, within four successive time periods 1968-1977, 1978-1987, 1988-1997 and 1998-2005. Cox regression analysis was used to investigate factors that may influence survival. Analyses were carried out separately by gender and age group (0-14, 15-24 years).

Results There were a total of 5917 cancer cases; 2958 aged 0-14 years (1659 males, 1299 females) and 2949 aged 15-24 years (1592 males, 1357 females). For childhood cancer (aged 0-14) five year survival rates for all cancers improved significantly $(P<0.0001)$ from $39 \%$ in $1968-1977$ to $60 \%$ in $1978-1987,75 \%$ in $1988-1997$ and $79 \%$ in 1998-2005. From the earliest to the latest period the survival rate for leukaemia increased from $24 \%$ to $81 \% \quad(P<0.0001)$, lymphoma from $46 \%$ to $87 \%(P<0.0001)$, central nervous system tumours (CNS) from $43 \%$ to $73 \%(P<0.0001)$, sympathetic nervous system tumours from $17 \%$ to $66 \%(P<0.0001)$, bone tumours from $21 \%$ to $75 \%(P<0.0001)$, soft tissue sarcoma from $30 \%$ to $58 \%$ $(P=0.0001)$ and for germ cell tumours from $59 \%$ to $97 \%(P=0.0002)$. Cox analysis showed worse survival for acute lymphocytic leukaemia (ALL) and astrocytoma in the age group 10-14 years. For cancer in teenage and young adults (aged 15-24) five year survival rates for all cancers improved from $47 \%$ in $1968-1977$ to $62 \%$ in $1978-1987,75 \%$ in $1988-1997$ and $83 \%$ in $1998-2005$. From the earliest to the latest period the survival rate for leukaemia increased from $2 \%$ to $57 \% \quad(P<0.0001)$, lymphoma from $66 \%$ to $87 \%$ $(P<0.0001)$, CNS tumours from $52 \%$ to $81 \% \quad(P=0.002)$, bone tumours from $35 \%$ to $55 \%$ ( $P=0.02$ ), germ cell tumours from $41 \%$ to 95\% $(P<0.0001)$ and carcinomas from $56 \%$ to $93 \%(P<0.0001)$. Survival was worse for ALL in the age group 20-24 years but better for non-Hodgkin lymphoma.

Conclusions There have been marked improvements in survival from childhood and young adult cancer in the North of England over the last four decades. Future work should analyse geographical and socio-demographic patterns for survival rates. 